\title{
Aviezri Fraenkel and Combinatorial Games
}

\author{
Richard K. Guy \\ Calgary, Canada
}

The subject of combinatorial games has, like combinatorics itself, been slow to find recognition with the mathematical establishment. Combinatorics is now on sure ground, and combinatorial games is well on its way. This is in no small part due to the energy, enthusiasm and insight of Aviezri Fraenkel, who has linked combinatorial games with much else in mathematics - graph theory [24], error-correcting codes [22], numeration systems [25, 28], continued fractions [17] and especially complexity theory.

The great variety in the difficulty of the vast range of combinatorial games has enabled him to exhibit the whole spectrum of complexity theory $[18,27,55]$, He has also ascertained the status of many particular combinatorial games [34], diophantine games [60], the Grundy function [14], chess [40], and checkers [33].

His interest in games may well have been sparked by a classic paper of Coxeter [6]: certainly he has long been fascinated by the relation between Beatty sequences, i.e. complementing sequences of integers, on the one hand, and Wythoff's Game $[9,39,11,1,8]$ on the other. Wythoff's Game is played with two heaps from which players alternately take any number from one heap or equal numbers from each; several of Aviezri's papers have been concerned with generalizations of this game [3, 7, 15, 17, 32, 45, 61].

He has written on a variety of individual games, many of which are his own invention: Nimbi [36], Nimhoff games [43], and Nim itself [4]; Geography [50], Epidemiography [41, 42, 44], geodetic contraction games [35], Particles and Antiparticles [12], a deletion game [48], a new heap game [58], modular Nim (sometimes called Kotzig's Nim) [37], Multivision [26], partizan octal and subtraction games [38] and extensions of Conway's 'short games' [52].

He has been especially interested in annihilation games $[10,53,54,56]$ and in games using Cedric Smith's extension of the Sprague-Grundy theory to cover games in which there are possible draws through infinite play $[2,371-375]$. He provided Conway with an early example [5]; other examples of his treatment of games with cycles are [46, 52] and the 'additional subtraction' games, where you may put as well as take [51].

A recent development is his adaptation of (one-player) cellular automata games to two players $[29,30,31]$. The motivation for this comes from his continuing interest in complexity theory, and the connections with linear error-correcting codes.

In addition to this remarkable output, he has served the combinatorial games fraternity in several important ways. In the organization of workshops, conferences and seminars he displayed 11 different games at the 1986 Strens Memorial Conference in Calgary [21], and was on the organizing committee of both the 1994 and 2000 workshops at MSRI. Berkeley, and there are other examples in the past and in the future. In stimulating dozens of co-workers - the publications listed below include more than 30 coauthors, besides his score of solo papers.. In writing general surveys [13, 20, 23]. In circulating 
problems, the life-blood of any mathematical discipline [16]. And, most importantly, in maintaining the definitive bibliography of the subject [19], which has also appeared in $[59,62]$ and will soon be updated in More Games of No Chance, the proceedings of the 2000 MSRI Workshop.

\section{References}

[1] Alexander Barabash, Aviezri S. Fraenkel \& Barak Weiss, Iterated Beatty functions, Random Comput. Dynamics, 1(1992/93) 333-348; MR 94g:11010.

[2] E. R. Berlekamp, J. H. Conway \& R. K. Guy, Winning Ways for your Mathematical Plays, Vol. I \& II, Academic Press, London, 1982; 2nd edition akpeters, 2001.

[3] Uri Blass \& Aviezri S. Fraenkel, The Sprague-Grundy function of Wythoff's game, Theoret. Comput. Sci. (Math Games), 75(1990), 311-333; MR 92a:90101.

[4] Uri Blass, Aviezri S. Fraenkel \& Romina Guelman, How far can Nim in disguise be stretched?, J. Combin. Theory Ser. A, 84(1998), 145-156; MR 2000d:91029.

[5] John Horton Conway, On Numbers and Games, Academic Press, 1976; 2nd edition akpeters, 2001, pp. 134-135.

[6] H. S. M. Coxeter, The golden section, phyllotaxis and Wythoff's game, Scripta Math., 19(1953) 135-143; MR 15-246b.

[7] N. Devduvani \& Aviezri S. Fraenkel, Properties of $k$-Welter's game, Discrete Math., 76(1989) 197-221; MR 91a:90182.

[8] Roger B. Eggleton, Aviezri S. Fraenkel \& R. Jamie Simpson, Beatty sequences and Langford sequences, Discrete Math., 111(1993) 165-178; MR 94a:11018.

[9] Aviezri S. Fraenkel, The bracket function and complementary sets of integers, Canad. J. Math., 21(1969) 6-27; MR 38 \#3214.

[10] Aviezri S. Fraenkel, Combinatorial games with an annihilation rule, in: J. P. LaSalle (ed.) The Influence of Computing on Mathematics Research and Education, Proc. Symp. Appl. Math., 20(1974), Amer. Math. Soc., 87-91; MR 50 \#6509.

[11] Aviezri S. Fraenkel, Complementary sets of integers, Amer. Math. Monthly, 84(1977) 114115; MR 55 \#2825.

[12] Aviezri S. Fraenkel, The particles and antiparticles game, Comput. Math. Appl., 3(1977), $327-328$.

[13] Aviezri S. Fraenkel, From Nim to Go, in J. Srivastava (ed.) Combinatorial Designs and Their Applications, Proc. Sympos. Combin. Math., Fort Collins CO (1978), Ann. Discrete Math., 6(1980) 137-156; MR 82e:90117. 
[14] Aviezri S. Fraenkel, Planar kernel and Grundy with $d \leq 3, d_{\text {out }} \leq 2, d_{\text {in }} \leq 2$ are NPcomplete Discrete Appl. Math. 3(1981), 257-262; MR 83j:68048.

[15] Aviezri S. Fraenkel, How to beat your Wythoff games' opponent on three fronts, Amer. Math. Monthly, 89(1982), 353-361.; MR 84k:90099

[16] Aviezri S. Fraenkel, 15 Research problems on games, Discrete Math., Research Problems section, 43-46(1983); MR 95c:05095.

[17] Aviezri S. Fraenkel, Wythoff games, continued fractions, cedar trees and Fibonacci searches, Theoret. Comput. Sci., 29(1984), 49-73; see also J. Diaz (ed.) Proc. 10th Internat. Colloq. Automata, Languages and Programming, Barcelona (1983), Lecture Notes Comput. Sci., 154(1983) 203-225; MR 85d:68030, 85k:68037.

[18] Aviezri S. Fraenkel, Complexity of games, in R. K. Guy (ed.) Combinatorial Games, Proc. Symp. Appl. Math., 43, Amer. Math. Soc., 1991, 111-153.

[19] Aviezri S. Fraenkel, Combinatorial games: selected bibliography with a succinct gourmet introduction, Electron. J. Combin., 1(1994) Dynamic Survey 2, 45pp. (electronic). \url\{http://www.combinatorics.org/Volume\_1/cover.html\}

[20] Aviezri S. Fraenkel, Recreation and depth in combinatorial games, in R. K. Guy \& R. E. Woodrow (eds.) The Lighter Side of Mathematics, Proc. Strens Memorial Conf. Recr. Math. and History, Calgary (1986), Math. Assoc. Amer. Spectrum Series, 1994, 159-173.

[21] Aviezri S. Fraenkel, Recreational games displays: combinatorial games, in R. K. Guy \& R. E. Woodrow (eds.) The Lighter Side of Mathematics, Proc. Strens Memorial Conf. Recr. Math. and History, Calgary (1986), Math. Assoc. Amer. Spectrum Series, 1994, 175-194.

[22] Aviezri S. Fraenkel, Error-correcting codes derived from combinatorial games, in R. J. Nowakowski (ed.) Games of No Chance, Proc. MSRI Workshop Combinatorial Games, Berkeley (1994), MSRI Publ. 29, Cambridge University Press, 1996, 417-431; MR 98h:94023.

[23] Aviezri S. Fraenkel, Scenic trails ascending from sea-level Nim to alpine chess, in: R. J. Nowakowski (ed.) Games of No Chance, Proc. MSRI Workshop Combinatorial Games, Berkeley (1994), MSRI Publ. 29, Cambridge University Press, 1996, 13-42; MR 98b:90195.

[24] Aviezri S. Fraenkel, Combinatorial game theory foundations applied to digraph kernels, Electron. J. Combin., 4(1997), \#R10, 17pp., Wilf Festschrift; MR 98d:05138.

\url\{http://www.combinatorics.org/Volume\_4/wilftoc.html\}

[25] Aviezri S. Fraenkel, Heap games, numeration systems and sequences, Annals Combin., 2(1998) 197-210; see also Fun With Algorithms, Vol. 4 of Proceedings in Informatics, Univ. Waterloo, Ont., 4(1998) 99-113.

[26] Aviezri S. Fraenkel, Multivision: an intractable impartial game with a linear winning strategy, Amer. Math. Monthly, 105(1998) 923-928. 
[27] Aviezri S. Fraenkel, Recent results and questions in combinatorial game complexities, in C. Iliopoulos (ed.) Proc. 9th Australasian Workshop Combin. Algorithms, Perth (1998), 124-146.

[28] Aviezri S. Fraenkel, Arrays, numeration systems and games, Ann. Combin., 2(1998) 197210; MR 2000b:91001.

[29] Aviezri S. Fraenkel, Mathematical chats between two physicists, in D. Wolfe \& T. Rodgers, eds., Puzzlers' Tribute: A Feast for the Mind, honoring Martin Gardner, A K Peters, 2001.

[30] Aviezri S. Fraenkel, Virus versus mankind, Proc. 2nd Internat. Conf. Computer Games (Hamamatsu, Japan, Oct. 2000) Lecture Notes in Computer Science, Springer, 2001.

[31] Aviezri S. Fraenkel, Two-player games on cellular automata, in R. J. Nowakowski, ed., More Games of No Chance, Proc. MSRI Workshop Combinatorial Games (Berkeley CA, 2000), Cambridge Univ. Press, 2002.

[32] Aviezri S. Fraenkel \& I. Borosh, A generalization of Wythoff's game, J. Combin. Theory Ser. A, 15(1973), 175-191; MR 49 \#4581.

[33] Aviezri S. Fraenkel, M. R. Garey, D. S. Johnson, T. Schaefer \& Yaacov Yesha, The complexity of checkers on an $n \times n$ board, Proc. 19th Symp. Foundations Comput. Sci., Ann Arbor (1978) 55-64.

[34] Aviezri S. Fraenkel \& Elisheva Goldschmidt, Pspace-hardness of some combinatorial games, J. Combin. Theory Ser. A, 46(1987) 21-38; MR 88j:68049.

[35] Aviezri S. Fraenkel \& Frank Harary, Geodetic contraction games on graphs, Internat. J. Game Theory. 18(1989), 327-338; MR 90m:90309.

[36] Aviezri S. Fraenkel \& Hans Herda, Never rush to be first in playing Nimbi, Math. Mag., 53(1980) 21-26; MR 82f:90101.

[37] Aviezri S. Fraenkel, Alan Jaffray, Anton Kotzig \& Gert Sabidussi, Modular Nim, Theoret. Comput. Sci., Math. Games, 143(1995), 319-333; MR 96f:90137.

[38] Aviezri S. Fraenkel \& Anton Kotzig, Partizan octal games: partizan subtraction games, Internat. J. Game Theory, 16(1987) 145-154; MR 88c:90145.

[39] Aviezri S. Fraenkel, Jonathan Levitt \& Michael Shimshoni, Characterization of the set of values $f(n)=\lfloor n \alpha\rfloor, n=1,2, \ldots$, Discrete Math., 2(1972) 335-345; MR 46 \#1743.

[40] Aviezri S. Fraenkel \& David Lichtenstein, Computing a perfect strategy for $n \times n$ chess requires time exponential in n, J. Combin. Theory Ser. A, 31(1981), 199-214; and in S. Even \& O. Kariv, (eds.) Proc. 8th Internat. Colloq. Automata, Language and Programming, Lecture Notes in Comput. Sci., 115(1981) 278-293; MR 83b:68044, 83c:90182.

[41] Aviezri S. Fraenkel, Martin Loebl \& Jaroslav Nešetřil, Epidemiography, II. Games with a dozing yet winning player, J. Combin. Theory Ser. A, 49(1988) 129-144; MR 90e:90170. 
[42] Aviezri S. Fraenkel \& Mordechai Lorberbom, Epidemiography with various growth functions Discrete Appl. Math., 25(1989), 53-71; MR 90m:90310.

[43] Aviezri S. Fraenkel \& Mordechai Lorberbom, Nimhoff games, J. Combin. Theory Ser. A, 58(1991), 1-25; MR 92i:90136.

[44] Aviezri S. Fraenkel \& Jaroslav Nešetřil, Epidemiography, Pacific J. Math., 118(1985) 369381; MR 87a:90152.

[45] Aviezri S. Fraenkel \& Michal Ozery, Adjoining to Wythoff's game its $P$-positions as moves, Theoret. Comput. Sci., 205(1998) 283-296; MR 99m:90185.

[46] Aviezri S. Fraenkel \& Y. Perl, Constructions in combinatorial games with cycles, in A. Hajnal, R. Rado \& V. T. Sós (eds.) Infinite and Finite Sets, Kesthely, (1973), Colloq. Math. Soc. János Bolyai 10(1975) Vol. 2 667-699,MR 52 \#5048.

[47] Aviezri S. Fraenkel \& Ofer Rahat, Infinite cyclic impartial games, Proc. 1st Internat. Conf. Computer Games, Tsukuba, Japan (1998), Lecture Notes in Comput. Sci., 1558(1999) 212-221; see also Theoret. Comput. Sci., 252(2001) 5-12.

[48] Aviezri S. Fraenkel \& Edward R. Scheinerman, A deletion game on hypergraphs, Discrete Appl. Math., 30(1991) 155-162.

[49] Aviezri S. Fraenkel, Edward R. Scheinerman \& Daniel Ullman, Undirected edge geography, Theoret. Comput. Sci., Math. Games, 112(1993), 371-381.

[50] Aviezri S. Fraenkel \& S. Simonson, Geography, Theoret. Comput. Sci. Math. Games, 110(1993), 197-214.

[51] Aviezri S. Fraenkel \& Uzi Tassa, Strategy for a class of games with dynamic ties, Comput. Math. Appl., 1(1975) 237-254; MR 54 \#2220.

[52] Aviezri S. Fraenkel \& Uzi Tassa, Strategies for compounds of partizan games, Math. Proc. Cambridge Philos. Soc., 92(1982) 193-204; MR 84k:90100.

[53] Aviezri S. Fraenkel, Uzi Tassa \& Yaacov Yesha, Three annihilation games, Math. Mag., 51(1978) 13-17; MR 58 \#15272.

[54] Aviezri S. Fraenkel \& Yaacov Yesha, Theory of annihilation games, Bull. Amer. Math. Soc., 82(1976) 775-777; MR 56 \#8027.

[55] Aviezri S. Fraenkel \& Yaacov Yesha, Complexity of problems in games, graphs and algebraic equations, Discrete Appl. Math., 1(1979) 15-30; MR 81c:90091.

[56] Aviezri S. Fraenkel \& Yaacov Yesha, Theory of annihilation games-I, J. Combin. Theory Ser. B, 33(1982) 60-86; MR 84c:90097.

[57] Aviezri S. Fraenkel \& Yaacov Yesha, The generalized Sprague-Grundy function and its invariance under certain mappings, J. Combin. Theory Ser. A, 43(1986) 165-177; MR 87m:90179. 
[58] Aviezri S. Fraenkel \& Dimitri Zusman, A new heap game, Proc. 1st Internat. Conf. Computer Games, Tsukuba, Japan (1998), Lecture Notes in Comput. Sci., 1558(1999) 205-211; see also Theoret. Comput. Sci., 252(2001) 5-12; MR 2000m:91027.

[59] Richard K. Guy (editor), Combinatorial Games, Proc. Sympos. Appl. Math., 43, Amer. Math. Soc., 1991, 191-226.

[60] James P. Jones \& Aviezri S. Fraenkel, Complexities of winning strategies in diophantine games, J. Complexity 11(1995) 435-455; MR 97c:68066.

[61] Joseph Kahane \& Aviezri S. Fraenkel, $k$-Welter - a generalization of Welter's game, $J$. Combin. Theory Ser. A 46(1987) 1-20; MR 88k:90221.

[62] Richard J. Nowakowski (editor), Games of No Chance: Combinatorial Games at MSRI, (1994), Cambridge Univ. Press, 1996, 493-537. 